\title{
Coherent functors in stable homotopy theory
}

\author{
by
}

Henning Krause (Bielefeld)

\begin{abstract}
Coherent functors $\mathcal{S} \rightarrow$ Ab from a compactly generated triangulated category into the category of abelian groups are studied. This is inspired by Auslander's classical analysis of coherent functors from a fixed abelian category into abelian groups. We characterize coherent functors and their filtered colimits in various ways. In addition, we investigate certain subcategories of $\mathcal{S}$ which arise from families of coherent functors.
\end{abstract}

Let $\mathcal{S}$ be a compactly generated triangulated category, for example the stable homotopy category of $\mathrm{CW}$-spectra. We call a functor $F: \mathcal{S} \rightarrow \mathrm{Ab}$ into the category of abelian groups coherent if there exists an exact sequence

$$
\operatorname{Hom}(D,-) \rightarrow \operatorname{Hom}(C,-) \rightarrow F \rightarrow 0
$$

such that $C$ and $D$ are compact objects in $\mathcal{S}$ (an object $X$ in $\mathcal{S}$ is compact if the representable functor $\operatorname{Hom}(X,-)$ preserves arbitrary coproducts).

The concept of a coherent functor has been introduced explicitly for abelian categories by Auslander [2], but it is also implicit in the work [9] of Freyd on stable homotopy. In this paper we characterize coherent functors in a number of ways and use them to study a wider class of functors $\mathcal{S} \rightarrow \mathrm{Ab}$ which share a weak exactness property. Another purpose of this paper is to investigate certain subcategories of $\mathcal{S}$ which are defined in terms of coherent functors.

In the category $\operatorname{Mod} \Lambda$ of modules over an associative ring $\Lambda$, the analogue of a compact object is a finitely presented module. This fact can be made precise (cf. the Appendix), and one has in this context the following classical result: a functor $F: \operatorname{Mod} \Lambda \rightarrow \mathrm{Ab}$ is coherent precisely if $F$ preserves products and filtered colimits. There is no obvious way to formulate such a characterization for compactly generated triangulated categories because filtered colimits rarely exist in triangulated categories. Nevertheless, we are able to characterize the coherent functors as follows.

2000 Mathematics Subject Classification: Primary 55U35; Secondary 18 E30. 
Theorem A. For a functor $F: \mathcal{S} \rightarrow$ Ab the following conditions are equivalent:

(1) $F$ is coherent.

(2) F preserves products and sends every homology colimit to a colimit.

(3) F preserves products and coproducts, and $F$ is short exact.

In the presence of Brown representability (for homology theories), there is a further equivalent condition:

(4) F preserves products and minimal weak filtered colimits of compact objects.

We call a functor $F: \mathcal{S} \rightarrow$ Ab short exact if for every triangle $X \rightarrow$ $Y \rightarrow Z \rightarrow \Sigma X$ the sequence $0 \rightarrow F(X) \rightarrow F(Y) \rightarrow F(Z) \rightarrow 0$ is exact, provided that $0 \rightarrow \operatorname{Hom}(C, X) \rightarrow \operatorname{Hom}(C, Y) \rightarrow \operatorname{Hom}(C, Z) \rightarrow 0$ is exact for each compact $C$. This seems to be an interesting exactness property. To explain this, let us introduce the following notation. The full subcategory of compact objects in $\mathcal{S}$ is denoted by $\mathcal{F}$, and $\left(\mathcal{F}^{\mathrm{op}}, \mathrm{Ab}\right)$ denotes the category of additive functors $\mathcal{F}^{\mathrm{op}} \rightarrow \mathrm{Ab}$ into the category of abelian groups. For every object $X$ in $\mathcal{S}$ consider the functor

$$
H_{X}=\left.\operatorname{Hom}(-, X)\right|_{\mathcal{F}}: \mathcal{F}^{\mathrm{op}} \rightarrow \mathrm{Ab} .
$$

This is an example of an exact functor. Recall that a functor from a triangulated category to the category of abelian groups is exact if it sends triangles to exact sequences. We call a triangle

$$
X \rightarrow Y \rightarrow Z \rightarrow \Sigma X
$$

in $\mathcal{S}$ pure if the induced sequence $0 \rightarrow H_{X} \rightarrow H_{Y} \rightarrow H_{Z} \rightarrow 0$ is exact, and a functor $F: \mathcal{S} \rightarrow \mathrm{Ab}$ is by definition short exact if for every pure triangle $(*)$ the sequence $0 \rightarrow F(X) \rightarrow F(Y) \rightarrow F(Z) \rightarrow 0$ is exact.

For the stable homotopy category, exact and coproduct preserving functors have been characterized by Brown and Adams [1]; they are precisely the functors $F: \mathcal{S} \rightarrow$ Ab that are "represented" by an object $Y$ in $\mathcal{S}$ in the sense that

$$
F(X) \cong \operatorname{Hom}(S, X \wedge Y)
$$

for all $X$ in $\mathcal{S}$ (where $S$ denotes the sphere spectrum). One can also use the tensor product

$$
\left(\mathcal{F}^{\mathrm{op}}, \mathrm{Ab}\right) \times(\mathcal{F}, \mathrm{Ab}) \rightarrow \mathrm{Ab}, \quad(H, G) \mapsto H \otimes_{\mathcal{F}} G,
$$

to study functors $\mathcal{S} \rightarrow$ Ab. Recall that the tensor functor $-\otimes_{\mathcal{F}} G$ is determined by the fact that it preserves colimits and $H_{X} \otimes_{\mathcal{F}} G \cong G(X)$ for all $X$ in $\mathcal{F}$. It turns out that $F: \mathcal{S} \rightarrow \mathrm{Ab}$ is exact and preserves coproducts if and only if there is a functorial isomorphism

$$
F(X) \cong H_{X} \otimes_{\mathcal{F}} G
$$


for some exact functor $G: \mathcal{F} \rightarrow$ Ab. The following result characterizes the functors which are "represented" by an arbitrary functor $G: \mathcal{F} \rightarrow$ Ab.

Theorem B. For a functor $F: \mathcal{S} \rightarrow$ Ab the following conditions are equivalent:

(1) $F$ is short exact and preserves coproducts.

(2) There exist an additive functor $G: \mathcal{F} \rightarrow \mathrm{Ab}$ and a functorial isomorphism $F(X) \cong H_{X} \otimes_{\mathcal{F}} G$ for all $X$ in $\mathcal{S}$.

(3) There exist a filtered diagram $\left(F_{i}\right)_{i \in \mathcal{I}}$ of coherent functors and a functorial isomorphism $F(X) \cong \operatorname{colim}_{i} F_{i}(X)$ for all $X$ in $\mathcal{S}$.

Consider the collection of all coherent functors $\mathcal{S} \rightarrow \mathrm{Ab}$, which we denote by $\operatorname{Coh} \mathcal{S}$. In fact, $\operatorname{Coh} \mathcal{S}$ is an abelian category if we take as maps the natural transformations. This category has been studied by Freyd in [9]. Here, we exhibit an interesting closure operation which is defined in terms of coherent functors. Given a class $\mathcal{C}$ of objects in $\mathcal{S}$, we define

$$
\operatorname{Def} \mathcal{C}=\{X \in \mathcal{S} \mid F(X)=0 \text { for all } F \in \operatorname{Coh} \mathcal{S} \text { with } F(\mathcal{C})=0\} .
$$

For example, Freyd's Generating Hypothesis [9] for the stable homotopy category could be reformulated as follows.

Generating Hypothesis (Freyd). $\operatorname{Def}\left\{S^{n} \mid n \in \mathbb{Z}\right\}=\mathcal{S}$.

There is an explicit construction which produces all objects in Def $\mathcal{C}$, at least if we assume Brown representability. We call an object $X$ the reduced product of a family of objects $\left(X_{i}\right)_{i \in I}$ in $\mathcal{S}$ with respect to a filter $\mathcal{U}$ on the set $I$ if

$$
H_{X} \cong \operatorname{colim}_{J \in \mathcal{U}} \prod_{i \in J} H_{X_{i}}
$$

where the filtered colimit is taken over the canonical projections $\prod_{i \in J_{1}} H_{X_{i}}$ $\rightarrow \prod_{i \in J_{2}} H_{X_{i}}$ which are induced by the inclusions $J_{2} \subseteq J_{1}$ of subsets $J_{1}, J_{2}$ $\in \mathcal{U}$. Note that a reduced product always exists; it is unique up to isomorphism and denoted by $\prod_{i \in I} X_{i} / \mathcal{U}$.

Theorem C. Suppose that Brown representability holds for $\mathcal{S}$, and let $\mathcal{C}$ be a class of objects in $\mathcal{S}$. Then an object $X$ in $\mathcal{S}$ belongs to Def $\mathcal{C}$ if and only if there is a pure triangle $X \rightarrow Y \rightarrow Z \rightarrow \Sigma X$ such that $Y$ is a reduced product of objects in $\mathcal{C}$.

We say that a full subcategory $\mathcal{C}$ of $\mathcal{S}$ is definable if $\mathcal{C}=\operatorname{Def} \mathcal{C}$, equivalently if $\mathcal{C}=\left\{X \in \mathcal{S} \mid F_{i}(X)=0\right.$ for all $\left.i \in I\right\}$ for some family $\left(F_{i}\right)_{i \in I}$ of coherent functors. This concept has its origin in model theory of modules; in this context a definable subcategory corresponds to a complete theory of modules $[23,8]$. There are three other concepts equivalent to definable subcategories: 
- Ziegler-closed subsets of the set $\operatorname{Sp} \mathcal{S}$ of isomorphism classes of indecomposable pure-injective objects in $\mathcal{S}$. Recall that $X$ in $\mathcal{S}$ is pure-injective if for every pure triangle $X \rightarrow Y \rightarrow Z \rightarrow \Sigma X$ the first map is a section. A subset of $\operatorname{Sp} \mathcal{S}$ is Ziegler-closed if it is of the form $\mathcal{C} \cap \operatorname{Sp} \mathcal{S}$ for some definable subcategory $\mathcal{C}$ of $\mathcal{S}$.

- Serre subcategories of Coh $\mathcal{S}$. These are full subcategories of Coh $\mathcal{S}$ which are closed under forming subobjects, quotient objects, and extensions.

- Cohomological ideals in $\mathcal{F}$. These are ideals of maps in $\mathcal{F}$ which are of the form $\{\phi \in \mathcal{F} \mid F(\phi)=0\}$ for some exact functor $F: \mathcal{F} \rightarrow$ Ab. For example, given $X$ in $\mathcal{S}$, the annihilator

$$
\text { Ann } X=\{\phi \in \mathcal{F} \mid \operatorname{Hom}(\phi, X)=0\}
$$

is cohomological.

Fundamental Correspondence. There are bijections between

- the set of definable subcategories $\mathcal{C}$ of $\mathcal{S}$,

- the set of Ziegler-closed subsets $\mathbf{U}$ of $\operatorname{Sp} \mathcal{S}$,

- the set of Serre subcategories $\mathcal{T}$ of $\operatorname{Coh} \mathcal{S}$,

- the set of cohomological ideals $\mathfrak{I}$ in $\mathcal{F}$.

These bijections are defined as follows:

$$
\begin{aligned}
& \mathcal{C}\left\{\begin{array}{l}
\mathbf{U}=\mathcal{C} \cap \operatorname{Sp} \mathcal{S}, \\
\mathcal{T}=\{F \in \operatorname{Coh} \mathcal{S} \mid F(X)=0 \text { for all } X \in \mathcal{C}\}, \\
\mathfrak{I}=\bigcap_{X \in \mathcal{C}} \text { Ann } X,
\end{array}\right. \\
& \mathbf{U} \mapsto\left\{\begin{array}{l}
\mathcal{C}=\left\{X \in \mathcal{S} \mid \text { there are } Y_{i} \in \mathbf{U} \text { and a pure mono } X \rightarrow \prod_{i} Y_{i}\right\}, \\
\mathcal{T}=\{F \in \operatorname{Coh} \mathcal{S} \mid F(X)=0 \text { for all } X \in \mathbf{U}\}, \\
\mathfrak{I}=\bigcap_{X \in \mathbf{U}} \text { Ann } X,
\end{array}\right. \\
& \mathcal{T} \mapsto\left\{\begin{array}{l}
\mathcal{C}=\{X \in \mathcal{S} \mid F(X)=0 \text { for all } F \in \mathcal{T}\}, \\
\mathbf{U}=\{X \in \operatorname{Sp} \mathcal{S} \mid F(X)=0 \text { for all } F \in \mathcal{T}\}, \\
\mathfrak{I}=\{\phi \in \mathcal{F} \mid \operatorname{Im} \operatorname{Hom}(\phi,-) \in \mathcal{T}\},
\end{array}\right. \\
& \mathfrak{I} \mapsto\left\{\begin{array}{l}
\mathcal{C}=\{X \in \mathcal{S} \mid \mathfrak{I} \subseteq \operatorname{Ann} X\}, \\
\mathbf{U}=\{X \in \operatorname{Sp} \mathcal{S} \mid \mathfrak{I} \subseteq \operatorname{Ann} X\}, \\
\mathcal{T}=\{F \in \operatorname{Coh} \mathcal{S} \mid F=\operatorname{Im} \operatorname{Hom}(\phi,-) \text { for some } \phi \in \mathfrak{I}\} .
\end{array}\right.
\end{aligned}
$$

This correspondence is the analogue of a correspondence for module categories which is based on work of several mathematicians [23, 12, 8, 15]. For instance, Ziegler introduced the closed subsets of indecomposable pure-injective modules in model-theoretic terms [23]. In our setting, one obtains a topology on $\operatorname{Sp} \mathcal{S}$ by taking the Ziegler-closed subsets as closed subsets [15]. Examples of definable subcategories arise quite naturally. Take for instance a localization functor $L: \mathcal{S} \rightarrow \mathcal{S}$ which is smashing, i.e. $L$ 
preserves coproducts. Then the $L$-local objects form a definable subcategory [17]. Or take an endofinite object $X$ in $\mathcal{S}$ (in the sense of [18]). Then the direct factors of coproducts of copies of $X$ form a definable subcategory.

\section{The functor category}

Purity and phantoms. We fix a triangulated category $\mathcal{S}$ and make the following additional assumptions:

- $\mathcal{S}$ has arbitrary coproducts;

- the isomorphism classes of compact objects in $\mathcal{S}$ form a set;

- $\operatorname{Hom}(C, X)=0$ for all compact $C$ implies $X=0$ for every object $X$ in $\mathcal{S}$.

A triangulated category satisfying these conditions is called compactly generated. The full subcategory of compact objects in $\mathcal{S}$ is always denoted by $\mathcal{F}$. Recall that $X$ in $\mathcal{S}$ is compact if the representable functor $\operatorname{Hom}(X,-)$ preserves arbitrary coproducts. Our basic tool is the category of additive functors $\mathcal{F}^{\mathrm{op}} \rightarrow \mathrm{Ab}$, which we denote by $\left(\mathcal{F}^{\mathrm{op}}, \mathrm{Ab}\right)$. The restricted Yoneda functor

$$
\mathcal{S} \rightarrow\left(\mathcal{F}^{\mathrm{op}}, \mathrm{Ab}\right), \quad X \mapsto H_{X}=\left.\operatorname{Hom}(-, X)\right|_{\mathcal{F}},
$$

relates the triangulated structure of $\mathcal{S}$ to the abelian structure of $\left(\mathcal{F}^{\mathrm{op}}, \mathrm{Ab}\right)$. The functor identifies the full subcategory of pure-projective objects in $\mathcal{S}$ with the full subcategory of projective objects in $\left(\mathcal{F}^{\mathrm{op}}, \mathrm{Ab}\right)$, and it identifies the full subcategory of pure-injective objects in $\mathcal{S}$ with the full subcategory of injective objects in $\left(\mathcal{F}^{\mathrm{op}}, \mathrm{Ab}\right)$. We briefly recall the relevant definitions and refer to [17] for more details.

DEFINITION 1.1. Let $\mathcal{S}$ be a compactly generated triangulated category.

(1) A map $X \rightarrow Y$ in $\mathcal{S}$ is a pure monomorphism if the map $H_{X} \rightarrow$ $H_{Y}$ is a monomorphism. An object $X$ in $\mathcal{S}$ is pure-injective if every pure monomorphism $X \rightarrow Y$ is a split monomorphism.

(2) A map $Y \rightarrow Z$ in $\mathcal{S}$ is a pure epimorphism if the map $H_{Y} \rightarrow H_{Z}$ is an epimorphism. An object $Z$ in $\mathcal{S}$ is pure-projective if every pure epimorphism $Y \rightarrow Z$ is a split epimorphism.

(3) A triangle $X \rightarrow Y \rightarrow Z \rightarrow \Sigma X$ is pure if the sequence $0 \rightarrow H_{X} \rightarrow$ $H_{Y} \rightarrow H_{Z} \rightarrow 0$ is exact.

Note that purity is closely related to properties of phantom maps; see for example [4] and [6]. Recall that a map $X \rightarrow Y$ in $\mathcal{S}$ is a phantom map if the induced map $H_{X} \rightarrow H_{Y}$ is zero. For instance, an object $X$ in $\mathcal{S}$ is pureinjective if and only if there are no non-zero phantom maps ending in $X$. Dually, $X$ is pure-projective if and only if there are no non-zero phantom maps starting in $X$. Finally, a triangle $X \rightarrow Y \rightarrow Z \rightarrow \Sigma X$ is pure if and only if the map $Z \rightarrow \Sigma X$ is phantom. 
One can prove easily that an object in $\mathcal{S}$ is pure-projective if and only if it is a direct factor of a coproduct of compact objects. The following lemma describes some essential properties of pure-projective objects. This is well known (see for example [4]), but we include the proof for the convenience of the reader.

Lemma 1.2. Let $\mathcal{S}$ be a compactly generated triangulated category, and let $P$ be a projective object in $\left(\mathcal{F}^{\mathrm{op}}, \mathrm{Ab}\right)$. Then there exists a unique (up to isomorphism) object $X$ in $\mathcal{S}$ such that $P \cong H_{X}$. Moreover, the map

$$
\operatorname{Hom}(X, Y) \rightarrow \operatorname{Hom}\left(H_{X}, H_{Y}\right), \quad \alpha \mapsto H_{\alpha},
$$

is an isomorphism for all $Y$ in $\mathcal{S}$.

Proof. Every projective $P$ is a direct factor of some coproduct $\coprod_{i \in I} H_{C_{i}}$ of representable functors with $C_{i} \in \mathcal{F}$ for all $i \in I$. Assume first that $P=$ $\coprod_{i \in I} H_{C_{i}}$. Then one takes $X=\coprod_{i \in I} C_{i}$ and the isomorphism $\operatorname{Hom}(X, Y) \cong$ $\operatorname{Hom}\left(H_{X}, H_{Y}\right)$ is an immediate consequence of Yoneda's lemma. The general case reduces to the first. In fact, if $P$ is a proper direct factor of $\coprod_{i \in I} H_{C_{i}}$, then we get a corresponding idempotent in $\operatorname{End}\left(\coprod_{i \in I} C_{i}\right)$ which gives an object $X$ in $\mathcal{S}$ satisfying $P \cong H_{X}$ since idempotents in $\mathcal{S}$ split.

The next lemma describes some properties of pure-injective objects. The proof is essentially an application of Brown's representability theorem.

Lemma 1.3. Let $\mathcal{S}$ be a compactly generated triangulated category, and let $I$ be an injective object in $\left(\mathcal{F}^{\mathrm{op}}, \mathrm{Ab}\right)$. Then there exists a unique (up to isomorphism) object $Y$ in $\mathcal{S}$ such that $I \cong H_{Y}$. Moreover, the map

$$
\operatorname{Hom}(X, Y) \rightarrow \operatorname{Hom}\left(H_{X}, H_{Y}\right), \quad \alpha \mapsto H_{\alpha},
$$

is an isomorphism for all $X$ in $\mathcal{S}$.

Proof. See Lemma 1.7 in [17].

Injective envelopes. We shall also need to use the fact that $\left(\mathcal{F}^{\mathrm{op}}, \mathrm{Ab}\right)$ is a Grothendieck category, which as far as we are concerned means that it has injective envelopes [11]. The definition of an injective envelope can be reformulated as follows.

Lemma 1.4. A monomorphism $\alpha: X \rightarrow Y$ is an injective envelope of $X$ if and only if $Y$ is an injective object and every endomorphism $\beta: Y \rightarrow Y$ satisfying $\beta \circ \alpha=\alpha$ is an isomorphism.

Brown representability. Sometimes we shall use an additional assumption on the category $\mathcal{S}$. To this end recall that a functor from a triangulated category to the category of abelian groups is exact if it sends triangles to exact sequences. For example, every functor of the form $H_{X}$ is exact. In some cases also the converse is true. More precisely, one says that Brown representability holds for $\mathcal{S}$ if 
- every exact functor $\mathcal{F}^{\mathrm{op}} \rightarrow \mathrm{Ab}$ is isomorphic to $H_{X}$ for some object $X$ in $\mathcal{S}$, and

- every natural transformation $H_{X} \rightarrow H_{Y}$ is of the form $H_{\alpha}$ for some map $\alpha: X \rightarrow Y$.

A classical theorem due to Brown and Adams states that Brown representability holds for the stable homotopy category [1]. More recently, Beligiannis, Christensen, Keller, and Neeman studied the problem of when Brown representability holds $[4,5]$.

Flat functors. Recall that there exists a tensor product

$$
\left(\mathcal{F}^{\mathrm{op}}, \mathrm{Ab}\right) \times(\mathcal{F}, \mathrm{Ab}) \rightarrow \mathrm{Ab}, \quad(F, G) \mapsto F \otimes_{\mathcal{F}} G
$$

where for any functor $F: \mathcal{F}^{\mathrm{op}} \rightarrow \mathrm{Ab}$, the tensor functor $F \otimes_{\mathcal{F}}$ - is determined by the fact that it preserves colimits and $F \otimes_{\mathcal{F}} \operatorname{Hom}(X,-) \cong F(X)$ for all $X$ in $\mathcal{F}$; see for example [20]. A functor $F: \mathcal{F}^{\text {op }} \rightarrow$ Ab is flat if the tensor functor $F \otimes_{\mathcal{F}}-$ is exact. The following well known characterization will be needed.

Lemma 1.5. For a functor $F: \mathcal{F}^{\mathrm{op}} \rightarrow \mathrm{Ab}$ the following are equivalent:

(1) $F$ is flat.

(2) $F$ is an exact functor.

(3) $F$ is a filtered colimit of representable functors.

Proof. For the characterization of flatness via condition (2), see Lemma 2.7 in [17]; for (3), see Theorem 3.2 in [21].

REMARK 1.6. The statement of Lemma 1.5 remains true if $\mathcal{F}$ is replaced by any skeletally small triangulated category.

Finitely presented functors. Some of our constructions involve finitely presented functors. Let us recall that a functor $F: \mathcal{F}^{\mathrm{op}} \rightarrow \mathrm{Ab}$ is finitely presented if there exists an exact sequence

$$
\operatorname{Hom}(-, C) \rightarrow \operatorname{Hom}(-, D) \rightarrow F \rightarrow 0
$$

with $C$ and $D$ in $\mathcal{F}$. We wish to distinguish bewteen finitely presented and coherent functors. Both are by definition cokernels of maps between representable functors. However, for coherent functors $\mathcal{S} \rightarrow$ Ab we restrict ourselves to representable functors which are represented by compact objects.

The concept of a finitely presented functor generalizes the concept of a finitely presented module, and we shall use a few basic facts about finitely presented functors which are well known in the context of modules over a ring. For instance, every additive functor $F: \mathcal{F}^{\mathrm{op}} \rightarrow \mathrm{Ab}$ is a filtered colimit of finitely presented functors. The following characterization is another example. 
LEMma 1.7. For an additive functor $F: \mathcal{F}^{\mathrm{op}} \rightarrow \mathrm{Ab}$ the following are equivalent:

(1) $F$ is finitely presented.

(2) The representable functor $\operatorname{Hom}(F,-)$ preserves filtered colimits.

(3) The tensor functor $F \otimes_{\mathcal{F}}-$ preserves products.

(4) The map $F \otimes_{\mathcal{F}} \prod_{i} \operatorname{Hom}\left(C_{i},-\right) \rightarrow \prod_{i}\left(F \otimes_{\mathcal{F}} \operatorname{Hom}\left(C_{i},-\right)\right)$ is an isomorphism for every family $\left(C_{i}\right)_{i \in I}$ in $\mathcal{F}$.

Proof. Adapt the proof for modules over a ring (cf. [22]).

2. Weak colimits. A diagram in a category $\mathcal{C}$ is a functor $\mathcal{I} \rightarrow \mathcal{C}$, $i \mapsto X_{i}$, from a small category $\mathcal{I}$ to $\mathcal{C}$. We denote such a diagram by $\left(X_{i}\right)_{i \in \mathcal{I}}$ and call a family of maps $\mu_{i}: X_{i} \rightarrow X(i \in \mathcal{I})$ a cone if $\mu_{j} \circ X_{\lambda}=\mu_{i}$ for every map $\lambda: i \rightarrow j$ in $\mathcal{I}$.

Definition 2.1. Let $\mu_{i}: X_{i} \rightarrow X(i \in \mathcal{I})$ be a cone of a diagram $\left(X_{i}\right)_{i \in \mathcal{I}}$.

(1) The cone is a weak colimit of the diagram $\left(X_{i}\right)_{i \in \mathcal{I}}$ if for every cone $\nu_{i}: X_{i} \rightarrow Y(i \in \mathcal{I})$ there exists a map $\alpha: X \rightarrow Y$ such that $\alpha \circ \mu_{i}=\nu_{i}$ for each $i \in \mathcal{I}$.

(2) The cone is minimal if every endomorphism $\alpha: X \rightarrow X$ satisfying $\alpha \circ \mu_{i}=\mu_{i}$ for each $i \in \mathcal{I}$ is an isomorphism.

If we require the factorization $\alpha: X \rightarrow Y$ in the definition of a weak colimit to be unique, this is the definition of a colimit, which we denote by $\operatorname{colim}_{i \in \mathcal{I}} X_{i}$. Note that every colimit is a minimal weak colimit. A minimal weak colimit of a diagram $\left(X_{i}\right)_{i \in \mathcal{I}}$ is unique up to a (non-unique) isomorphism. Our terminology is borrowed from Auslander [3]. He calls a map $\alpha: X \rightarrow Y$ left minimal if every endomorphism $\beta: Y \rightarrow Y$ satisfying $\beta \circ \alpha=\alpha$ is an isomorphism. Viewing a cone of a diagram $\mathcal{I} \rightarrow \mathcal{C}$ as a map in the category of all functors $\mathcal{I} \rightarrow \mathcal{C}$, it is clear that this map is left minimal if and only if the cone is minimal.

DEFinition 2.2. Let $\left(X_{i}\right)_{i \in \mathcal{I}}$ be a diagram in a compactly generated triangulated category $\mathcal{S}$. A cone $X_{i} \rightarrow X(i \in \mathcal{I})$ is called a homology colimit of the diagram $\left(X_{i}\right)_{i \in \mathcal{I}}$ if the induced map $\operatorname{colim}_{i \in \mathcal{I}} \operatorname{Hom}\left(C, X_{i}\right) \rightarrow$ $\operatorname{Hom}(C, X)$ is an isomorphism for every compact object $C$ in $\mathcal{S}$.

Note that a homology colimit of a diagram $\left(X_{i}\right)_{i \in \mathcal{I}}$ is minimal and therefore unique up to a (non-unique) isomorphism; it is denoted by hcolim $i \in \mathcal{I} X_{i}$. Our terminology is justified by the following observation.

Proposition 2.3. A cone $X_{i} \rightarrow X(i \in \mathcal{I})$ is a homology colimit if and only if for every exact and coproduct preserving functor $H: \mathcal{S} \rightarrow \mathrm{Ab}$ the induced map $\operatorname{colim}_{i} H\left(X_{i}\right) \rightarrow H(X)$ is an isomorphism. 
Proof. One direction is clear. Therefore suppose that the cone $X_{i} \rightarrow X$ $(i \in \mathcal{I})$ is a homology colimit and fix an exact and coproduct preserving functor $H: \mathcal{S} \rightarrow \mathrm{Ab}$. The restriction $\left.H\right|_{\mathcal{F}}$ is exact and therefore it is a filtered colimit of representable functors by Lemma 1.5, that is,

$$
\left.\left.\operatorname{colim}_{j \in \mathcal{J}} \operatorname{Hom}\left(C_{j},-\right)\right|_{\mathcal{F}} \cong H\right|_{\mathcal{F}} .
$$

We obtain a filtered diagram of representable functors $\left(\operatorname{Hom}\left(C_{j},-\right)\right)_{j \in \mathcal{J}}$ and a compatible set of maps $\operatorname{Hom}\left(C_{j},-\right) \rightarrow H(j \in \mathcal{J})$, using Yoneda's lemma. This induces a functorial isomorphism $\operatorname{colim}_{j \in \mathcal{J}} \operatorname{Hom}\left(C_{j}, X\right) \cong H(X)$ for all $X$ in $\mathcal{S}$ because both sides are exact, agree on $\mathcal{F}$, and preserve coproducts (cf. [17, Proposition 3.2]). We obtain the following commutative diagram:

$$
\begin{aligned}
& \operatorname{colim}_{i} H\left(X_{i}\right) \cong \operatorname{colim}_{i} \operatorname{colim}_{j} \operatorname{Hom}\left(C_{j}, X_{i}\right) \cong \operatorname{colim}_{j} \operatorname{colim}_{i} \operatorname{Hom}\left(C_{j}, X_{i}\right) \\
& \downarrow \text { c } \\
& \downarrow \beta \\
& H(X) \cong \operatorname{colim}_{j} \operatorname{Hom}\left(C_{j}, X\right)=\operatorname{colim}_{j} \operatorname{Hom}\left(C_{j}, X\right)
\end{aligned}
$$

The map $\gamma$ is the colimit of isomorphisms by our assumption on the cone, and we conclude that $\alpha$ is an isomorphism.

In [19], Margolis discusses weak colimits for the stable homotopy category, and there is also a more recent treatment in [13]. However, the definitions of a minimal weak colimit in [19] and [13] are more restrictive than the one given here. Note that our Proposition 2.3 generalizes Proposition 2.2.2 of [13].

Given a diagram $\left(X_{i}\right)_{i \in \mathcal{I}}$ in a compactly generated triangulated category, a weak colimit always exists. In fact, a weak colimit can be computed by taking the cofiber of an appropriate map $\coprod_{\lambda: i \rightarrow j} X_{i} \rightarrow \coprod_{k} X_{k}$ where $\lambda: i \rightarrow j$ runs through all maps and $k$ runs through all objects in $\mathcal{I}$. The following result is essentially due to Margolis [19], and closely related to Theorem 4.2.3 of $[13]$.

Proposition 2.4. Let $\mathcal{S}$ be a compactly generated triangulated category and suppose that Brown representability holds. Then every filtered diagram of pure-projective objects in $\mathcal{S}$ has a homology colimit which is also a minimal weak colimit.

Proof. Let $\left(X_{i}\right)_{i \in \mathcal{I}}$ be a filtered diagram of pure-projective objects. The functor $\operatorname{colim}_{i} H_{X_{i}}$ is exact and therefore isomorphic to $H_{X}$ for some $X$ in $\mathcal{S}$ since we assume Brown representability. Using Lemma 1.2 and the fact that each $X_{i}$ is pure-projective, we get a family of maps $X_{i} \rightarrow X(i \in \mathcal{I})$ which is a cone for $\left(X_{i}\right)_{i \in \mathcal{I}}$. Moreover, this cone is a homology colimit by construction. In order to show that the cone is a weak colimit, let $X_{i} \rightarrow Y$ $(i \in \mathcal{I})$ be another cone. By Brown representability again, the induced map $H_{X} \cong \operatorname{colim}_{i} H_{X_{i}} \rightarrow H_{Y}$ is of the form $H_{\alpha}$ for some $\alpha: X \rightarrow Y$. The map $\alpha$ 
is compatible with the structural maps $X_{i} \rightarrow X$ and $X_{i} \rightarrow Y$ by Lemma 1.2, and therefore $X_{i} \rightarrow X(i \in \mathcal{I})$ is a weak colimit.

Next we collect a few basic facts about the existence of minimal weak colimits for arbitrary diagrams.

Lemma 2.5. Let $\left(X_{i}\right)_{i \in \mathcal{I}}$ be a diagram in an additive category $\mathcal{C}$ and suppose that idempotents in $\mathcal{C}$ split. Let $X_{i} \rightarrow X(i \in \mathcal{I})$ be a weak colimit and denote by $M$ the image of the induced map $\operatorname{Hom}(X, X) \rightarrow \prod_{i} \operatorname{Hom}\left(X_{i}, X\right)$. Then the following conditions are equivalent:

(1) The $\operatorname{End}(X)$-module $M$ has a projective cover.

(2) The diagram $\left(X_{i}\right)_{i \in \mathcal{I}}$ has a minimal weak colimit.

Moreover, in this case $X_{i} \rightarrow X(i \in \mathcal{I})$ is minimal if and only if the canonical map $\operatorname{Hom}(X, X) \rightarrow M$ is a projective cover.

Proof. The proof is straightforward if one observes that an epimorphism $\pi: P \rightarrow M$ with $P$ projective is a projective cover of $M$ if and only if every endomorphism $\varepsilon: P \rightarrow P$ satisfying $\pi \circ \varepsilon=\pi$ is an isomorphism.

Let $X$ be an object in an additive category and suppose that idempotents split. Then every finitely generated $\operatorname{End}(X)$-module has a projective cover if and only if $X$ decomposes into finitely many indecomposable objects with local endomorphism rings. Using this elementary fact, one can prove the following.

Proposition 2.6. Every finite diagram of compact objects in the category of p-local spectra has a minimal weak colimit.

Proof. Every compact $p$-local spectrum $X$ decomposes into finitely many indecomposable objects with local endomorphism rings (cf. [10]). The assertion is therefore a consequence of Lemma 2.5 because every finitely generated $\operatorname{End}(X)$-module has a projective cover.

Another method to produce minimal weak colimits is to construct appropriate injective envelopes.

Proposition 2.7. A diagram in a compactly generated triangulated category has a minimal weak colimit provided there exists a weak colimit which is pure-injective.

Proof. Let $\mu_{i}: X_{i} \rightarrow X(i \in \mathcal{I})$ be a cone of some diagram and suppose that $X$ is pure-injective. It follows from Lemma 1.3 and the characterization of injective envelopes in Lemma 1.4 that the cone is minimal if and only if for $\mu: \coprod_{i} X_{i} \rightarrow X$ the induced map $\operatorname{Im} H_{\mu} \rightarrow H_{X}$ is an injective envelope. Now suppose that the above cone is a weak colimit. Taking an injective envelope $\operatorname{Im} H_{\mu} \rightarrow H_{Y}$ produces a new cone $\mu_{i}: X_{i} \rightarrow Y(i \in \mathcal{I})$ which is a minimal weak colimit. 
We end this section with a characterization of pure triangles. Let us call a triangle $X \rightarrow Y \rightarrow Z \rightarrow \Sigma X$ split if the identity map $\operatorname{id}_{Z}$ factors through $Y \rightarrow Z$, equivalently, if $\operatorname{id}_{X}$ factors through $X \rightarrow Y$.

Lemma 2.8. The following are equivalent for a triangle $\delta: X \rightarrow Y \rightarrow$ $Z \rightarrow \Sigma X$ in a compactly generated triangulated category:

(1) $\delta$ is pure.

(2) $\delta$ is a homology colimit of a filtered diagram of split triangles of compact objects.

(3) $\delta$ is a homology colimit of a filtered diagram of split triangles.

Proof. $(1) \Rightarrow(2)$. Given an object $X$ in $\mathcal{S}$, the functor $H_{X}: \mathcal{F}^{\mathrm{op}} \rightarrow \mathrm{Ab}$ is exact and therefore flat by Lemma 1.5. A well known consequence of this is the fact that the category $\mathcal{I}_{X}$ whose objects are the maps $X_{i} \rightarrow X$ with $X_{i}$ compact and whose maps are the obvious commuting triangles forms a small filtered category with hcolim ${ }_{i \in \mathcal{I}_{X}} X_{i}=X$ (cf. [21, Theorem 3.2]). Analogously, one shows that for any map $\beta: Y \rightarrow Z$ in $\mathcal{S}$ the category $\mathcal{I}_{\beta}$ whose objects are the commuting squares

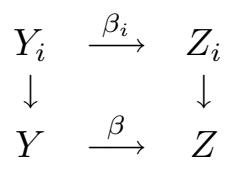

with $Y_{i}$ and $Z_{i}$ compact and whose maps $i \rightarrow j$ are the obvious commuting squares

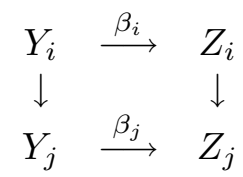

forms a small filtered category with hcolim $\operatorname{li\mathcal {I}}_{\beta} \beta_{i}=\beta$.

Now suppose that the triangle $\delta: X \stackrel{\alpha}{\longrightarrow} Y \stackrel{\beta}{\longrightarrow} Z \stackrel{\gamma}{\longrightarrow} \Sigma X$ is pure. It follows that the commuting squares $i \in \mathcal{I}_{\beta}$ with $\beta_{i}$ a split epimorphism form a cofinal subcategory of $\mathcal{I}_{\beta}$ which we denote by $\mathcal{I}$. In fact, every commuting square $i \in \mathcal{I}_{\beta}$ fits into a commutative diagram of the form

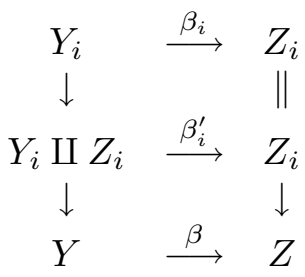

where the second component of $\beta_{i}^{\prime}$ is the identity since the map $Z_{i} \rightarrow Z$ factors through $\beta$. We obtain a filtered diagram $\left(\delta_{i}\right)_{i \in \mathcal{I}}$ of split triangles $\delta_{i}$ : $X_{i} \stackrel{\alpha_{i}}{\longrightarrow} Y_{i} \stackrel{\beta_{i}}{\longrightarrow} Z_{i} \stackrel{\gamma_{i}}{\longrightarrow} \Sigma X_{i}$ and one easily checks that the commuting squares 
corresponding to the $\alpha_{i}$ and $\gamma_{i}$ with $i \in \mathcal{I}$ form cofinal subcategories of $\mathcal{I}_{\alpha}$ and $\mathcal{I}_{\gamma}$, respectively. We conclude that $\operatorname{hcolim}_{i \in \mathcal{I}} \alpha_{i}=\alpha, \operatorname{hcolim}_{i \in \mathcal{I}} \beta_{i}=\beta$, and $\operatorname{hcolim}_{i \in \mathcal{I}} \gamma_{i}=\gamma$. Thus $\delta=\operatorname{hcolim}_{i \in \mathcal{I}} \delta_{i}$.

$(2) \Rightarrow(3)$. Clear.

$(3) \Rightarrow(1)$. Suppose that $\delta=\operatorname{hcolim}_{i} \delta_{i}$ and that each $\delta_{i}$ is split. A split triangle $\delta_{i}: X_{i} \rightarrow Y_{i} \rightarrow Z_{i} \rightarrow \Sigma X_{i}$ induces an exact sequence $0 \rightarrow H_{X_{i}} \rightarrow$ $H_{Y_{i}} \rightarrow H_{Z_{i}} \rightarrow 0$. Taking filtered homology colimits preserves exactness and we get therefore an exact sequence $0 \rightarrow H_{X} \rightarrow H_{Y} \rightarrow H_{Z} \rightarrow 0$. Thus $\delta$ is pure.

3. Weak limits. The concept of a (minimal) weak limit is the obvious analogue of a (minimal) weak colimit which one obtains by reversing all the arrows in Definition 2.1. In this section we investigate the existence of minimal weak limits. We need the following lemma.

LEMmA 3.1. Let $X \stackrel{\alpha}{\longrightarrow} I \stackrel{\beta}{\longrightarrow} J \stackrel{\gamma}{\longrightarrow} \Sigma X$ be a triangle in $\mathcal{S}$ and suppose that the induced map $\operatorname{Im} H_{\beta} \rightarrow H_{J}$ is an injective envelope. Then every endomorphism $\varepsilon: X \rightarrow X$ satisfying $\alpha \circ \varepsilon=\alpha$ is an isomorphism.

Proof. Choose a map $\phi: J \rightarrow J$ which completes the following commutative diagram:

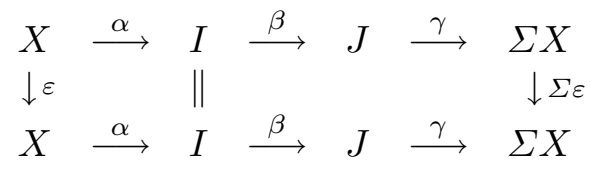

The assumption on $\varepsilon$ implies that $H_{\phi}$ keeps $\operatorname{Im} H_{\beta}$ fixed, and therefore $H_{\phi}$ is an isomorphism by Lemma 1.4. Thus $\phi$ is an isomorphism, and we conclude that $\varepsilon$ is an isomorphism.

We are now in a position to prove an existence criterion for minimal weak limits.

THEOREM 3.2. Let $\mathcal{S}$ be a compactly generated triangulated category. Then every diagram of pure-injective objects in $\mathcal{S}$ has a minimal weak limit.

Proof. The proof uses the fact that the category $\left(\mathcal{F}^{\mathrm{op}}, \mathrm{Ab}\right)$ has injective envelopes. Let $\left(X_{i}\right)_{i \in \mathcal{I}}$ be a diagram of pure-injective objects in $\mathcal{S}$ and let $F=\lim _{i} H_{X_{i}}$ be the corresponding limit in $\left(\mathcal{F}^{\mathrm{op}}, \mathrm{Ab}\right)$. There exists a minimal injective copresentation of $F$ which is of the form

$$
0 \rightarrow F \rightarrow H_{I} \stackrel{H_{\beta}}{\longrightarrow} H_{J}
$$

by Lemma 1.3. We complete $\beta$ to a triangle $X \stackrel{\alpha}{\longrightarrow} I \stackrel{\beta}{\longrightarrow} J \stackrel{\gamma}{\longrightarrow} \Sigma X$. The map $H_{\alpha}$ induces a map $H_{X} \rightarrow F$ which we compose with the structural maps $F \rightarrow H_{X_{i}}$ to obtain a family of maps $\mu_{i}: X \rightarrow X_{i}$, using Lemma 1.3 and the fact that each $X_{i}$ is pure-injective. We claim that $\mu_{i}: X \rightarrow X_{i}(i \in \mathcal{I})$ is a 
minimal weak limit of the diagram $\left(X_{i}\right)_{i \in \mathcal{I}}$. Observe first that $X_{\lambda} \circ \mu_{i}=\mu_{j}$ for every map $\lambda: i \rightarrow j$ in $\mathcal{I}$ since $H_{X_{\lambda}} \circ H_{\mu_{i}}=H_{\mu_{j}}$, again by Lemma 1.3. Now suppose there is another family $\nu_{i}: Y \rightarrow X_{i}(i \in \mathcal{I})$ of maps satisfying $X_{\lambda} \circ \nu_{i}=\nu_{j}$ for every map $\lambda: i \rightarrow j$ in $\mathcal{I}$. The family $H_{Y} \rightarrow H_{X_{i}}(i \in \mathcal{I})$ induces a map $H_{Y} \rightarrow F$ which we compose with $F \rightarrow H_{I}$ to get a map $Y \rightarrow I$. The composition of this map with $\beta$ is zero and therefore $Y \rightarrow I$ factors through $\alpha$ via some map $Y \rightarrow X$. Using again the pure-injectivity of the $X_{i}$, it is easy to check that $Y \rightarrow X$ is compatible with the strucural maps $\mu_{i}$ and $\nu_{i}$.

It remains to show that the family $X \rightarrow X_{i}(i \in \mathcal{I})$ is minimal. Every endomorphism $\varepsilon: X \rightarrow X$ which is compatible with the $\mu_{i}$ induces a map $H_{\varepsilon}$ which is compatible with the map $H_{X} \rightarrow F$. Therefore $\alpha \circ \varepsilon=\alpha$, and Lemma 3.1 implies that $\varepsilon$ is an isomorphism. This shows that the weak limit is minimal.

4. Extending functors. Suppose there is given a functor $F: \mathcal{S} \rightarrow \mathrm{Ab}$. It is often useful to extend $F$ to a functor $\hat{F}:\left(\mathcal{F}^{\mathrm{op}}, \mathrm{Ab}\right) \rightarrow$ Ab such that $\hat{F}\left(H_{X}\right)=F(X)$ for all $X$ in $\mathcal{S}$. We consider a number of conditions on $F$ which translate into properties of the functor $\hat{F}$.

(E) $\quad F$ is short exact, that is, for every pure triangle $X \rightarrow Y \rightarrow Z \rightarrow \Sigma X$ in $\mathcal{S}$ the sequence $0 \rightarrow F(X) \rightarrow F(Y) \rightarrow F(Z) \rightarrow 0$ is exact.

(I) $\quad F\left(\prod_{i} X_{i}\right) \cong \prod_{i} F\left(X_{i}\right)$ for every family $\left(X_{i}\right)_{i \in I}$ of pure-injective objects in $\mathcal{S}$.

( $\Sigma) \quad \coprod_{i} F\left(X_{i}\right) \cong F\left(\coprod_{i} X_{i}\right)$ for every family $\left(X_{i}\right)_{i \in I}$ of pure-projective objects in $\mathcal{S}$.

It is sometimes convenient to work with the following variants of (П) and $(\Sigma)$, respectively:

$\left(\Pi^{\prime}\right) \quad F\left(\prod_{i} X_{i}\right) \cong \prod_{i} F\left(X_{i}\right)$ for every family $\left(X_{i}\right)_{i \in I}$ of compact objects in $\mathcal{S}$.

$\left(\Sigma^{\prime}\right) \quad \coprod_{i} F\left(X_{i}\right) \cong F\left(\coprod_{i} X_{i}\right)$ for every family $\left(X_{i}\right)_{i \in I}$ of compact objects in $\mathcal{S}$.

Note that $\left(\Sigma^{\prime}\right)$ and $(\Sigma)$ are equivalent since every pure-projective object is a direct factor of a coproduct of compact objects. It turns out that condition (E) is sufficient to construct a functor $\hat{F}$ which extends $F$.

Proposition 4.1. Let $\mathcal{S}$ be a compactly generated triangulated category and let $F: \mathcal{S} \rightarrow \mathrm{Ab}$ be a short exact functor. Then there exists a unique (up to isomorphism) functor $\hat{F}:\left(\mathcal{F}^{\mathrm{op}}, \mathrm{Ab}\right) \rightarrow \mathrm{Ab}$ which is left exact and extends $F$, that is, $\hat{F}\left(H_{X}\right)=F(X)$ for all $X$ in $\mathcal{S}$. Moreover, 
(1) if $F$ satisfies $(\Pi)$, then $\hat{F}$ preserves products, and

(2) if $F$ satisfies $(\Sigma)$, then $\hat{F}$ preserves filtered colimits.

Proof. For every object $X$ in $\left(\mathcal{F}^{\mathrm{op}}, \mathrm{Ab}\right)$, choose an injective copresentation

$$
0 \rightarrow X \rightarrow H_{I} \stackrel{H_{\alpha}}{\longrightarrow} H_{J}
$$

This is possible by Lemma 1.3. Now one defines $\hat{F}(X)=\operatorname{Ker} F(\alpha)$ and checks easily that this can be extended to maps in $\left(\mathcal{F}^{\mathrm{op}}, \mathrm{Ab}\right)$ and that it is well defined. Condition $(\mathrm{E})$ implies that $\hat{F}\left(H_{X}\right)=F(X)$ for all $X$ in $\mathcal{S}$. In fact, we can choose for $X$ pure triangles

$$
X \rightarrow I_{0} \rightarrow X_{1} \rightarrow \Sigma X \quad \text { and } \quad X_{1} \rightarrow I_{1} \rightarrow X_{2} \rightarrow \Sigma X_{1}
$$

with $I_{i}$ pure-injective. This gives an injective copresentation $0 \rightarrow H_{X} \rightarrow$ $H_{I_{0}} \rightarrow H_{I_{1}}$, and $F(X)=\hat{F}\left(H_{X}\right)$ follows since $0 \rightarrow F(X) \rightarrow F\left(I_{0}\right) \rightarrow F\left(I_{1}\right)$ is exact. Clearly, $\hat{F}$ is left exact by construction. Moreover, any left exact functor $\left(\mathcal{F}^{\mathrm{op}}, \mathrm{Ab}\right) \rightarrow \mathrm{Ab}$ is uniquely determined by its restriction to the full subcategory of injective objects.

Suppose now that (ח) holds. This condition says that the restriction of $\hat{F}$ to the full subcategory of injectives in $\left(\mathcal{F}^{\mathrm{op}}, \mathrm{Ab}\right)$ preserves products. Let $\left(X_{i}\right)_{i \in I}$ be a family of arbitrary objects in $\left(\mathcal{F}^{\mathrm{op}}, \mathrm{Ab}\right)$ and choose injective copresentations $0 \rightarrow X_{i} \rightarrow I_{i} \rightarrow J_{i}$. We get the following commutative diagram with exact rows since $\hat{F}$ is left exact:

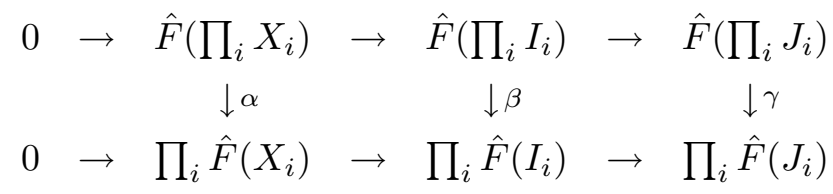

The maps $\beta$ and $\gamma$ are isomorphisms and it follows that $\alpha$ is an isomorphism. Thus $\hat{F}$ preserves products.

Finally suppose that $(\Sigma)$ holds. We construct a new functor $\check{F}:\left(\mathcal{F}^{\mathrm{op}}, \mathrm{Ab}\right)$ $\rightarrow \mathrm{Ab}$ as follows. For a finitely presented functor $X$ in $\left(\mathcal{F}^{\mathrm{op}}, \mathrm{Ab}\right)$ choose a presentation

$$
\operatorname{Hom}(-, A) \stackrel{\operatorname{Hom}(-, \alpha)}{\longrightarrow} \operatorname{Hom}(-, B) \rightarrow X \rightarrow 0
$$

and complete $\alpha$ to a triangle $A \stackrel{\alpha}{\longrightarrow} B \stackrel{\beta}{\longrightarrow} C \stackrel{\gamma}{\longrightarrow} \Sigma A$. We get an exact sequence

$$
0 \rightarrow X \rightarrow \operatorname{Hom}(-, C) \stackrel{\operatorname{Hom}(-, \gamma)}{\longrightarrow} \operatorname{Hom}(-, \Sigma A) .
$$

Now define $\check{F}(X)=\operatorname{Ker} F(\gamma)$. Every object $X$ in $\left(\mathcal{F}^{\text {op }}, \mathrm{Ab}\right)$ can be written as a filtered colimit of finitely presented functors. More precisely, the category $\mathcal{I}_{X}$ whose objects are the maps $X_{i} \rightarrow X$ with $X_{i}$ finitely presented and whose maps are the obvious commuting triangles forms a small filtered category with $\operatorname{colim}_{i \in \mathcal{I}_{X}} X_{i}=X$. One defines $\check{F}(X)=\operatorname{colim}_{i} \check{F}\left(X_{i}\right)$ and checks easily that this definition can be extended to maps in $\left(\mathcal{F}^{\mathrm{op}}, \mathrm{Ab}\right)$. Clearly, 
$\check{F}$ preserves filtered colimits. We claim that $\check{F}$ is left exact. To see this, fix an exact sequence $0 \rightarrow X \stackrel{\alpha}{\longrightarrow} Y \stackrel{\beta}{\longrightarrow} Z$ in $\left(\mathcal{F}^{\text {op }}, \mathrm{Ab}\right)$ and write $\beta=\operatorname{colim}_{i} \beta_{i}$ as a filtered colimit of maps $\beta_{i}: Y_{i} \rightarrow Z_{i}$ between finitely presented objects. We get a filtered diagram of exact sequences $0 \rightarrow X_{i} \stackrel{\alpha_{i}}{\longrightarrow} Y_{i} \stackrel{\beta_{i}}{\longrightarrow} Z_{i}$ and $0 \rightarrow \check{F}\left(X_{i}\right) \stackrel{\check{F}\left(\alpha_{i}\right)}{\longrightarrow} \check{F}\left(Y_{i}\right) \stackrel{\check{F}\left(\beta_{i}\right)}{\longrightarrow} \check{F}\left(Z_{i}\right)$ is exact by construction. Taking filtered colimits preserves exactness and it follows that $0 \rightarrow \check{F}(X) \stackrel{\check{F}(\alpha)}{\longrightarrow}$ $\check{F}(Y) \stackrel{\check{F}(\beta)}{\longrightarrow} \check{F}(Z)$ is exact.

Next we use $(\mathrm{E})$ and $(\Sigma)$ to show that $\check{F}\left(H_{X}\right)=F(X)$ for every $X$ in $\mathcal{S}$. Condition $(\Sigma)$ implies that this holds if $X$ is pure-projective. Otherwise choose for $X=X_{0}$ pure triangles

$$
X_{2} \rightarrow P_{1} \rightarrow X_{1} \rightarrow \Sigma X_{2} \quad \text { and } \quad X_{1} \rightarrow P_{0} \rightarrow X_{0} \rightarrow \Sigma X_{1}
$$

with $P_{i}$ pure-projective. Each sequence $0 \rightarrow H_{X_{i+1}} \rightarrow H_{P_{i}} \rightarrow H_{X_{i}} \rightarrow 0$ is a filtered colimit of split exact sequences of the form $0 \rightarrow H_{A} \rightarrow H_{B} \rightarrow H_{C}$ $\rightarrow 0$ with $A, B, C$ compact, by Lemma 2.8 . Thus $0 \rightarrow \check{F}\left(H_{X_{i+1}}\right) \rightarrow \check{F}\left(H_{P_{i}}\right)$ $\rightarrow \check{F}\left(H_{X_{i}}\right) \rightarrow 0$ is exact and therefore $\check{F}\left(H_{P_{1}}\right) \rightarrow \check{F}\left(H_{P_{0}}\right) \rightarrow \check{F}\left(H_{X}\right) \rightarrow 0$ is exact. On the other hand, $F\left(P_{1}\right) \rightarrow F\left(P_{0}\right) \rightarrow F(X) \rightarrow 0$ is exact by (E), and therefore $\check{F}\left(H_{X}\right)=F(X)$. It follows that $\check{F}$ and $\hat{F}$ are isomorphic, and therefore $\hat{F}$ preserves filtered colimits.

The preceding proposition has an analogue for functors $\left(\mathcal{F}^{\mathrm{op}}, \mathrm{Ab}\right) \rightarrow \mathrm{Ab}$ which are right exact and extend a functor $F: \mathcal{S} \rightarrow \mathrm{Ab}$. The construction uses projective presentations instead of injective copresentations.

Proposition 4.2. Let $\mathcal{S}$ be a compactly generated triangulated category and let $F: \mathcal{S} \rightarrow \mathrm{Ab}$ be a short exact functor. Then there exists a unique (up to isomorphism) functor $\check{F}:\left(\mathcal{F}^{\mathrm{op}}, \mathrm{Ab}\right) \rightarrow \mathrm{Ab}$ which is right exact and extends $F$, that is, $F\left(H_{X}\right)=F(X)$ for all $X$ in $\mathcal{S}$. Moreover, if $F$ satisfies $(\Sigma)$, then $\check{F}$ preserves coproducts and is isomorphic to $-\otimes_{\mathcal{F}} G$ where $G=$ $\left.F\right|_{\mathcal{F}}$.

Proof. The proof is analogous to that of Proposition 4.1 and we leave the details to the reader. The last assertion about $-\otimes_{\mathcal{F}} G$ follows from the fact that $\check{F}\left(H_{C}\right)=F(C)=H_{C} \otimes_{\mathcal{F}} G$ for each $C \in \mathcal{F}$ and every $X \in\left(\mathcal{F}^{\mathrm{op}}, \mathrm{Ab}\right)$ has a presentation

$$
\coprod_{j} H_{D_{j}} \rightarrow \coprod_{i} H_{C_{i}} \rightarrow X \rightarrow 0
$$

with $C_{i}, D_{j} \in \mathcal{F}$ for all $i, j$.

5. Coherent functors. We are now in a position to prove the first portion of our characterization of coherent functors. 
Proposition 5.1. Let $\mathcal{S}$ be a compactly generated triangulated category. For a functor $F: \mathcal{S} \rightarrow \mathrm{Ab}$ the following conditions are equivalent:

(1) $F$ is coherent.

(2) F preserves products and sends every homology colimit to a colimit.

(3) $F$ preserves products and coproducts, and $F$ is short exact.

(4) F satisfies (E), ( $\Pi)$, and $(\Sigma)$.

Proof. $(1) \Rightarrow(2)$. Each representable functor $\operatorname{Hom}(C,-)$ with $C$ compact preserves products and sends every homology colimit to a colimit by the definition of a homology colimit. Clearly, this property is preserved if we pass to the cokernel of a map $\operatorname{Hom}(D,-) \rightarrow \operatorname{Hom}(C,-)$. Thus (2) holds for every coherent functor $F$.

$(2) \Rightarrow(3)$. Suppose that $F$ preserves homology colimits. It follows that $F$ preserves coproducts because every coproduct in $\mathcal{S}$ is a homology colimit. Now suppose that $\delta: X \rightarrow Y \rightarrow Z \rightarrow \Sigma X$ is a pure triangle. It has been shown in Lemma 2.8 that $\delta$ is a homology colimit of split triangles $\delta_{i}: X_{i} \rightarrow Y_{i} \rightarrow Z_{i} \rightarrow \Sigma X_{i}$. Clearly, each sequence $0 \rightarrow F\left(X_{i}\right) \rightarrow$ $F\left(Y_{i}\right) \rightarrow F\left(Z_{i}\right) \rightarrow 0$ is exact since $F$ is additive, and therefore the colimit $0 \rightarrow \operatorname{colim}_{i} F\left(X_{i}\right) \rightarrow \operatorname{colim}_{i} F\left(Y_{i}\right) \rightarrow \operatorname{colim}_{i} F\left(Z_{i}\right) \rightarrow 0$ is exact. However, this sequence is isomorphic to $0 \rightarrow F(X) \rightarrow F(Y) \rightarrow F(Z) \rightarrow 0$ by our assumption on $F$. This proves (3).

$(3) \Rightarrow(4)$. Clear.

$(4) \Rightarrow(1)$. We apply Proposition 4.1 to get a functor $\hat{F}:\left(\mathcal{F}^{\mathrm{op}}, \mathrm{Ab}\right) \rightarrow \mathrm{Ab}$ which is left exact and extends $F$. Moreover, $(\Pi)$ and $(\Sigma)$ imply that $\hat{F}$ preserves products and filtered colimits. It follows that $\hat{F}$ preserves limits since every limit can be computed by taking kernels and products. Therefore the Adjoint Functor Theorem implies the existence of a left adjoint $G: \mathrm{Ab} \rightarrow$ $\left(\mathcal{F}^{\mathrm{op}}, \mathrm{Ab}\right)$ for $\hat{F}$. This gives for $X$ in $\left(\mathcal{F}^{\mathrm{op}}, \mathrm{Ab}\right)$ a functorial isomorphism

$$
\hat{F}(X) \cong \operatorname{Hom}(\mathbb{Z}, \hat{F}(X)) \cong \operatorname{Hom}(G(\mathbb{Z}), X) .
$$

The criterion of Lemma 1.7 implies that $G(\mathbb{Z})$ is a finitely presented functor since $\hat{F}$ preserves filtered colimits. Choose a presentation

$$
\operatorname{Hom}(-, A) \stackrel{\operatorname{Hom}(-, \alpha)}{\longrightarrow} \operatorname{Hom}(-, B) \rightarrow G(\mathbb{Z}) \rightarrow 0 .
$$

Applying $\operatorname{Hom}\left(-, H_{X}\right)$ gives an exact sequence

$$
0 \rightarrow \operatorname{Hom}\left(G(\mathbb{Z}), H_{X}\right) \rightarrow \operatorname{Hom}\left(\operatorname{Hom}(-, B), H_{X}\right) \rightarrow \operatorname{Hom}\left(\operatorname{Hom}(-, A), H_{X}\right),
$$

which is isomorphic to

$$
0 \rightarrow F(X) \rightarrow \operatorname{Hom}(B, X) \stackrel{\operatorname{Hom}(\alpha, X)}{\longrightarrow} \operatorname{Hom}(A, X)
$$

thanks to Yoneda's lemma and the isomorphism $F(X) \cong \operatorname{Hom}\left(G(\mathbb{Z}), H_{X}\right)$. This sequence is functorial in $X$, and if we complete $\alpha: A \rightarrow B$ to a triangle 
$A \rightarrow B \rightarrow C \rightarrow \Sigma A$, we get the desired presentation

$$
\operatorname{Hom}(\Sigma A,-) \rightarrow \operatorname{Hom}(C,-) \rightarrow F \rightarrow 0,
$$

which shows that $F$ is coherent.

Proposition 5.2. Let $\mathcal{S}$ be a compactly generated triangulated category. For a functor $F: \mathcal{S} \rightarrow \mathrm{Ab}$ the following conditions are equivalent:

(1) $F$ is coherent.

(2) $F$ satisfies $(\mathrm{E}),\left(\Pi^{\prime}\right)$, and $\left(\Sigma^{\prime}\right)$.

Proof. $(1) \Rightarrow(2)$ is shown in Proposition 5.1. Therefore suppose that $F$ satisfies $(\mathrm{E}),\left(\Pi^{\prime}\right)$, and $\left(\Sigma^{\prime}\right)$. Using conditions $(\mathrm{E})$ and $\left(\Sigma^{\prime}\right)$, we can apply Proposition 4.2 and extend $F$ to a functor $\check{F}:\left(\mathcal{F}^{\mathrm{op}}, \mathrm{Ab}\right) \rightarrow$ Ab which is isomorphic to $-\otimes_{\mathcal{F}} G$ for $G=\left.F\right|_{\mathcal{F}}$. In particular, $F(X) \cong H_{X} \otimes_{\mathcal{F}} G$ for all $X \in \mathcal{S}$. We claim that $G$ is finitely presented. In fact, this follows from Lemma 1.7 and condition $\left(\Pi^{\prime}\right)$ since for every family $\left(C_{i}\right)_{i \in I}$ in $\mathcal{F}$ we have

$$
\left(\prod_{i} H_{C_{i}}\right) \otimes_{\mathcal{F}} G \cong H_{\prod_{i} C_{i}} \otimes_{\mathcal{F}} G \cong F\left(\prod_{i} C_{i}\right) \cong \prod_{i} F\left(C_{i}\right) \cong \prod_{i}\left(H_{C_{i}} \otimes_{\mathcal{F}} G\right) .
$$

Tensoring a presentation $\operatorname{Hom}(D,-) \rightarrow \operatorname{Hom}(C,-) \rightarrow G \rightarrow 0$ with $H_{X}$ for $X \in \mathcal{S}$ gives an exact sequence

$$
H_{X} \otimes_{\mathcal{F}} \operatorname{Hom}(D,-) \rightarrow H_{X} \otimes_{\mathcal{F}} \operatorname{Hom}(C,-) \rightarrow H_{X} \otimes_{\mathcal{F}} G \rightarrow 0,
$$

which is isomorphic to

$$
\operatorname{Hom}(D, X) \rightarrow \operatorname{Hom}(C, X) \rightarrow F(X) \rightarrow 0 .
$$

This sequence is functorial in $X$ and therefore $F$ is coherent.

The next proposition completes our characterization of coherent functors.

Proposition 5.3. Let $\mathcal{S}$ be a compactly generated triangulated category and suppose that Brown representability holds. For a functor $F: \mathcal{S} \rightarrow \mathrm{Ab}$ the following conditions are equivalent:

(1) $F$ is coherent.

(2) $F$ preserves products of families $\left(X_{i}\right)_{i \in I}$ and minimal weak colimits of filtered diagrams $\left(X_{j}\right)_{j \in \mathcal{J}}$ provided that each $X_{i}$ and each $X_{j}$ is a direct factor of a coproduct of compact objects.

(3) $F$ preserves products of families $\left(X_{i}\right)_{i \in I}$ and minimal weak colimits of filtered diagrams $\left(X_{j}\right)_{j \in \mathcal{J}}$ provided that each $X_{i}$ and each $X_{j}$ is a compact object.

(4) $F$ satisfies $(\mathrm{E}),\left(\Pi^{\prime}\right)$, and $\left(\Sigma^{\prime}\right)$.

Proof. $(1) \Rightarrow(2)$. A coherent functor preserves products and sends homology colimits to colimits by Proposition 5.1. Every minimal weak colimit of 
a filtered diagram of pure-projective objects in $\mathcal{S}$ is also a homology colimit by Proposition 2.4. Therefore (1) implies (2).

$(2) \Rightarrow(3)$. Clear.

$(3) \Rightarrow(4)$. We check that $F$ satisfies the conditions $(\mathrm{E})$ and $\left(\Sigma^{\prime}\right)$.

(E) It has been shown in Lemma 2.8 that a pure triangle $\delta: X \rightarrow Y \rightarrow$ $Z \rightarrow \Sigma X$ can be expressed as a homology colimit of a diagram $\left(\delta_{i}\right)_{i \in \mathcal{I}}$ of split triangles $\delta_{i}: X_{i} \rightarrow Y_{i} \rightarrow Z_{i} \rightarrow \Sigma X_{i}$ of compact objects. Note that $\delta$ is also a minimal weak colimit of the diagram $\left(\delta_{i}\right)_{i \in \mathcal{I}}$ by Proposition 2.4. Applying $F$ gives a filtered diagram of exact sequences $0 \rightarrow F\left(X_{i}\right) \rightarrow F\left(Y_{i}\right) \rightarrow F\left(Z_{i}\right) \rightarrow 0$. The colimit of these exact sequences is again exact and isomorphic to the sequence $0 \rightarrow F(X) \rightarrow F(Y) \rightarrow F(Z) \rightarrow 0$, by our assumptions on $F$.

$\left(\Sigma^{\prime}\right)$ The coproduct of a family $\left(X_{i}\right)_{i \in I}$ is the filtered colimit of the finite coproducts $\coprod_{i \in J} X_{i}$ where $J$ runs through all finite subsets of $I$. Note that $\coprod_{i \in J} X_{i}=\prod_{i \in J} X_{i}$ if $J$ is finite. Thus (3) implies that $F$ preserves coproducts of compact objects in $\mathcal{S}$.

$(4) \Rightarrow(1)$. See Proposition 5.2.

6. Short exact functors. In this section we study some properties of short exact functors. Recall that a functor $F: \mathcal{S} \rightarrow \mathrm{Ab}$ is short exact if for every triangle $X \rightarrow Y \rightarrow Z \rightarrow \Sigma X$ the sequence $0 \rightarrow F(X) \rightarrow$ $F(Y) \rightarrow F(Z) \rightarrow 0$ is exact whenever $0 \rightarrow \operatorname{Hom}(C, X) \rightarrow \operatorname{Hom}(C, Y) \rightarrow$ $\operatorname{Hom}(C, Z) \rightarrow 0$ is exact for every compact $C$.

TheOREM 6.1. For a functor $F: \mathcal{S} \rightarrow$ Ab the following conditions are equivalent:

(1) $F$ is short exact and preserves coproducts.

(2) There exists an additive functor $G: \mathcal{F} \rightarrow \mathrm{Ab}$ and a functorial isomorphism $F(X) \cong H_{X} \otimes_{\mathcal{F}} G$ for all $X$ in $\mathcal{S}$.

(3) There exists a filtered diagram $\left(F_{i}\right)_{i \in \mathcal{I}}$ of coherent functors and a functorial isomorphism $F(X) \cong \operatorname{colim}_{i} F_{i}(X)$ for all $X$ in $\mathcal{S}$.

Proof. $(1) \Rightarrow(2)$. Let $G=\left.F\right|_{\mathcal{F}}$. Condition (1) implies that $F$ extends to a functor $\check{F}:\left(\mathcal{F}^{\mathrm{op}}, \mathrm{Ab}\right) \rightarrow \mathrm{Ab}$ which is isomorphic to $-\otimes_{\mathcal{F}} G$ by Proposition 4.2. Therefore

for all $X$ in $\mathcal{S}$.

$$
F(X) \cong \check{F}\left(H_{X}\right) \cong H_{X} \otimes_{\mathcal{F}} G
$$

$(2) \Rightarrow(3)$. Suppose that $F(X) \cong H_{X} \otimes_{\mathcal{F}} G$ for some functor $G: \mathcal{F} \rightarrow$ Ab. Writing $G=\operatorname{colim}_{i} G_{i}$ as a filtered colimit of finitely presented functors, we get a filtered diagram of coherent functors $F_{i}: \mathcal{S} \rightarrow$ Ab if we define $F_{i}(X)=H_{X} \otimes_{\mathcal{F}} G_{i}$ for each $i$. This gives an isomorphism

$$
F(X) \cong H_{X} \otimes_{\mathcal{F}}\left(\operatorname{colim}_{i} G_{i}\right) \cong \operatorname{colim}_{i}\left(H_{X} \otimes_{\mathcal{F}} G_{i}\right)=\operatorname{colim}_{i} F_{i}(X)
$$

since $H_{X} \otimes_{\mathcal{F}}-$ preserves colimits. 
$(3) \Rightarrow(1)$. A coherent functor is short exact and preserves coproducts by Proposition 5.1. Taking filtered colimits preserves exactness and coproducts, and therefore a filtered colimit of coherent functors is short exact and preserves coproducts.

A short exact functor kills phantom maps, and we have the converse if the functor is exact.

Theorem 6.2. Let $F: \mathcal{S} \rightarrow \mathrm{Ab}$ be a functor.

(1) If $F$ is short exact, then $F(\alpha)=0$ for every phantom map $\alpha$.

(2) Suppose $F$ is exact. Then $F$ is short exact if and only if $F(\alpha)=0$ for every phantom map $\alpha$.

Proof. Apply Proposition 4.1.

It is not true in general that a functor which kills phantom maps is short exact. Take for instance an object $Z$ in $\mathcal{S}$ which is not pure-projective, and let $F(X)=\operatorname{Hom}\left(H_{Z}, H_{X}\right)$ for $X$ in $\mathcal{S}$. Clearly, $F(\alpha)=0$ for every phantom map $\alpha$. However, if $X \rightarrow Y \rightarrow Z \rightarrow \Sigma X$ is a pure triangle with $Y$ pure-projective, the sequence $0 \rightarrow F(X) \rightarrow F(Y) \rightarrow F(Z) \rightarrow 0$ cannot be exact.

7. Definable subcategories. In this section we use coherent functors to study certain subcategories of $\mathcal{S}$.

Definition 7.1. We call a full subcategory $\mathcal{C}$ of $\mathcal{S}$ definable if it is of the form

$$
\mathcal{C}=\left\{X \in \mathcal{S} \mid F_{i}(X)=0 \text { for all } i \in I\right\}
$$

for some family $\left(F_{i}\right)_{i \in I}$ of coherent functors.

There are three other concepts equivalent to definable subcategories:

- Ziegler-closed subsets of the set $\operatorname{Sp} \mathcal{S}$ of indecomposable pure-injectives in $\mathcal{S}$,

- Serre subcategories of $\operatorname{Coh} \mathcal{S}$, and

- cohomological ideals in $\mathcal{F}$.

We refer to the introduction for precise definitions and the statement of the "fundamental correspondence" which relates these concepts to each other. Here, we use the functor category $\left(\mathcal{F}^{\mathrm{op}}, \mathrm{Ab}\right)$ to prove this correspondence. We start with some preparations.

Let $\operatorname{fp}\left(\mathcal{F}^{\mathrm{op}}, \mathrm{Ab}\right)$ be the full subcategory formed by the finitely presented functors in $\left(\mathcal{F}^{\mathrm{op}}, \mathrm{Ab}\right)$. Note that $\mathrm{fp}\left(\mathcal{F}^{\mathrm{op}}, \mathrm{Ab}\right)$ is abelian since $\mathcal{F}$ has weak kernels. Given a functor $F: \mathcal{F}^{\mathrm{op}} \rightarrow \mathrm{Ab}$, we define a functor $F^{\vee}: \mathcal{S} \rightarrow$ Ab by

$$
F^{\vee}(X)=\operatorname{Hom}\left(F, H_{X}\right) .
$$


LEMmA 7.2. The assignment $F \mapsto F^{\vee}$ induces an equivalence

$$
\left(\mathrm{fp}\left(\mathcal{F}^{\mathrm{op}}, \mathrm{Ab}\right)\right)^{\mathrm{op}} \rightarrow \mathrm{Coh} \mathcal{S} .
$$

Proof. Let $F \in \operatorname{fp}\left(\mathcal{F}^{\mathrm{op}}, \mathrm{Ab}\right)$ and fix a presentation

$$
\operatorname{Hom}(-, A) \rightarrow \operatorname{Hom}(-, B) \rightarrow F \rightarrow 0 .
$$

Complete the map $A \rightarrow B$ to a triangle $A \rightarrow B \rightarrow C \rightarrow \Sigma A$. It follows from Yoneda's lemma that we get a presentation

$$
\operatorname{Hom}(\Sigma A,-) \rightarrow \operatorname{Hom}(C,-) \rightarrow F^{\vee} \rightarrow 0 .
$$

Thus $F^{\vee}$ is coherent. It is now straightforward to write down an inverse for $F \mapsto F^{\vee}$.

We denote by $\operatorname{Spec}\left(\mathcal{F}^{\mathrm{op}}, \mathrm{Ab}\right)$ the set of isomorphism classes of indecomposable injective objects in $\left(\mathcal{F}^{\mathrm{op}}, \mathrm{Ab}\right)$. A subset of $\operatorname{Spec}\left(\mathcal{F}^{\mathrm{op}}, \mathrm{Ab}\right)$ is closed if it is of the form $\left\{X \in \operatorname{Spec}\left(\mathcal{F}^{\text {op }}, \mathrm{Ab}\right) \mid \operatorname{Hom}\left(F_{i}, X\right)=0\right.$ for all $\left.i \in I\right\}$ for some family $\left(F_{i}\right)_{i \in I}$ of finitely presented functors $\mathcal{F}^{\mathrm{op}} \rightarrow \mathrm{Ab}$.

Proposition 7.3. The assignments

$$
\begin{aligned}
& \mathbf{U} \mapsto\left\{F \in \operatorname{fp}\left(\mathcal{F}^{\mathrm{op}}, \mathrm{Ab}\right) \mid \operatorname{Hom}(F, X)=0 \text { for all } X \in \mathbf{U}\right\}, \\
& \mathcal{T} \mapsto\left\{X \in \operatorname{Spec}\left(\mathcal{F}^{\mathrm{op}}, \mathrm{Ab}\right) \mid \operatorname{Hom}(F, X)=0 \text { for all } F \in \mathcal{T}\right\}
\end{aligned}
$$

induce mutually inverse bijections between the closed subsets of the set $\operatorname{Spec}\left(\mathcal{F}^{\mathrm{op}}, \mathrm{Ab}\right)$ and the Serre subcategories of $\operatorname{fp}\left(\mathcal{F}^{\mathrm{op}}, \mathrm{Ab}\right)$.

Proof. See Theorem 4.2 in [15].

Given an object $X$ in $\mathcal{S}$, we consider the annihilator

$$
\text { Ann } X=\{\phi \in \mathcal{F} \mid \operatorname{Hom}(\phi, X)=0\} .
$$

Clearly, Ann $X$ is a cohomological ideal in $\mathcal{F}$, and the converse is also true.

Proposition 7.4. Every cohomological ideal in $\mathcal{F}$ is of the form Ann $X$ for some pure-injective object $X$ in $\mathcal{S}$.

Proof. We fix a cohomological ideal I. By definition, there exists an exact functor $F: \mathcal{F} \rightarrow$ Ab such that $\mathfrak{I}=\{\phi \in \mathcal{F} \mid F(\phi)=0\}$. The functor $-\otimes_{\mathcal{F}} F:\left(\mathcal{F}^{\mathrm{op}}, \mathrm{Ab}\right) \rightarrow \mathrm{Ab}$ is exact by Lemma 1.5 , and we obtain therefore a Serre subcategory of $\operatorname{fp}\left(\mathcal{F}^{\mathrm{op}}, \mathrm{Ab}\right)$ by taking

$$
\mathcal{T}=\left\{G \in \operatorname{fp}\left(\mathcal{F}^{\mathrm{op}}, \mathrm{Ab}\right) \mid G \otimes_{\mathcal{F}} F=0\right\} .
$$

Now let $I$ be the product of all $Y \in \operatorname{Spec}\left(\mathcal{F}^{\mathrm{op}}, \mathrm{Ab}\right) \operatorname{such}$ that $\operatorname{Hom}(G, Y)=0$ for all $G \in \mathcal{T}$. The correspondence in Proposition 7.3 implies

$$
\mathcal{T}=\left\{G \in \operatorname{fp}\left(\mathcal{F}^{\mathrm{op}}, \mathrm{Ab}\right) \mid \operatorname{Hom}(G, I)=0\right\},
$$


and we find $X \in \mathcal{S}$ with $H_{X} \cong I$ by Lemma 1.3. Now let $\phi$ be an arbitrary map in $\mathcal{F}$ and put $G=\operatorname{Im} H_{\phi}$. We get

$$
\begin{aligned}
F(\phi)=0 & \Leftrightarrow G \otimes_{\mathcal{F}} F=0 \Leftrightarrow \operatorname{Hom}\left(G, H_{X}\right)=0 \Leftrightarrow \operatorname{Hom}(\phi, X)=0 \\
& \Leftrightarrow \phi \in \operatorname{Ann} X .
\end{aligned}
$$

Thus $\mathfrak{I}$ is of the form Ann $X$.

Proof of the Fundamental Correspondence. The assignment $X \mapsto H_{X}$ identifies the pure-injective objects in $\mathcal{S}$ with the injective objects in the category $\left(\mathcal{F}^{\mathrm{op}}, \mathrm{Ab}\right)$ (cf. [17, Corollary 1.9]) and induces therefore a bijection $\operatorname{Sp} \mathcal{S} \rightarrow \operatorname{Spec}\left(\mathcal{F}^{\mathrm{op}}, \mathrm{Ab}\right)$ which identifies the Ziegler-closed subsets of $\operatorname{Sp} \mathcal{S}$ with the closed subsets of $\operatorname{Spec}\left(\mathcal{F}^{\mathrm{op}}, \mathrm{Ab}\right)$ by Lemma 7.2. We conclude from Proposition 7.3 that

$$
\begin{aligned}
& \mathbf{U} \mapsto\{F \in \operatorname{Coh} \mathcal{S} \mid F(X)=0 \text { for all } X \in \mathbf{U}\}, \\
& \mathcal{T} \mapsto\{X \in \operatorname{Sp} \mathcal{S} \mid F(X)=0 \text { for all } F \in \mathcal{T}\}
\end{aligned}
$$

induce mutually inverse bijections between Ziegler-closed subsets of $\operatorname{Sp} \mathcal{S}$ and Serre subcategories of Coh $\mathcal{S}$. It is an immediate consequence that

$$
\begin{aligned}
\mathcal{C} & \mapsto F \in \operatorname{Coh} \mathcal{S} \mid F(X)=0 \text { for all } X \in \mathcal{C}\}, \\
\mathcal{T} & \mapsto\{X \in \mathcal{S} \mid F(X)=0 \text { for all } F \in \mathcal{T}\}
\end{aligned}
$$

induce mutually inverse bijections between definable subcategories of $\mathcal{S}$ and Serre subcategories of Coh $\mathcal{S}$. In other words: a definable subcategory $\mathcal{C}$ is already determined by $\mathcal{C} \cap \operatorname{Sp} \mathcal{S}$. In fact, each definable subcategory $\mathcal{C}$ can be reconstructed explicitly from the corresponding Ziegler-closed subset $\mathbf{U}=\mathcal{C} \cap \operatorname{Sp} \mathcal{S}$ since

$\mathcal{C}=\left\{X \in \mathcal{S} \mid\right.$ there are $Y_{i} \in \mathbf{U}$ and a pure triangle $\left.X \rightarrow \prod_{i} Y_{i} \rightarrow Z \rightarrow \Sigma X\right\}$.

This follows from Proposition 3.2 of [15].

Next we consider the cohomological ideals. Note that a functor $F: \mathcal{S} \rightarrow \mathrm{Ab}$ is coherent precisely if $F=\operatorname{Im} \operatorname{Hom}(\phi,-)$ for some map $\phi: C \rightarrow D$ in $\mathcal{F}$. Clearly, $F(X)=0$ for some $X$ in $\mathcal{S}$ if and only if $\phi \in$ Ann $X$. By the correspondence between definable subcategories and Serre subcategories of coherent functors, it follows that $\mathcal{C} \mapsto \bigcap_{X \in \mathcal{C}}$ Ann $X$ induces an injective map from the set of definable subcategories of $\mathcal{S}$ into the set of cohomological ideals in $\mathcal{F}$. It remains to show that this map is surjective. To this end fix a cohomological ideal $\mathfrak{I}$ in $\mathcal{F}$. We have $\mathfrak{I}=\operatorname{Ann} Y$ for some $Y \in \mathcal{S}$ by Proposition 7.4. Thus $\mathcal{C}=\{X \in \mathcal{S} \mid \mathfrak{I} \subseteq$ Ann $X\}$ is a definable subcategory satisfying

$$
\mathfrak{I}=\bigcap_{X \in \mathcal{C}} \operatorname{Ann} X
$$


This completes the proof of the correspondence between definable subcategories, Ziegler-closed subsets, Serre subcategories and cohomological ideals.

Given a class $\mathcal{C}$ of objects in $\mathcal{S}$, the definable subcategory generated by $\mathcal{C}$ is

$$
\operatorname{Def} \mathcal{C}=\{X \in \mathcal{S} \mid F(X)=0 \text { for all } F \in \operatorname{Coh} \mathcal{S} \text { with } F(\mathcal{C})=0\} .
$$

It remains to prove the following description of Def $\mathcal{C}$ via reduced products which is formulated in Theorem C.

TheOREM 7.5. Suppose that Brown representability holds for $\mathcal{S}$, and let $\mathcal{C}$ be a class of objects in $\mathcal{S}$. Then an object $X$ in $\mathcal{S}$ belongs to Def $\mathcal{C}$ if and only if there is a pure triangle $X \rightarrow Y \rightarrow Z \rightarrow \Sigma X$ such that $Y=$ $\prod_{i \in I} X_{i} / \mathcal{U}$ for some family $\left(X_{i}\right)_{i \in I}$ of objects in $\mathcal{C}$ and some filter $\mathcal{U}$ on $I$.

Proof. We fix a class $\mathcal{C}$ of objects in $\mathcal{S}$ and put

$$
\mathcal{T}=\{F \in \operatorname{Coh} \mathcal{S} \mid F(X)=0 \text { for all } X \in \mathcal{C}\} .
$$

We again use the functor category $\left(\mathcal{F}^{\mathrm{op}}, \mathrm{Ab}\right)$. Recall that $F \in\left(\mathcal{F}^{\mathrm{op}}, \mathrm{Ab}\right)$ is $f p$-injective if $\operatorname{Ext}^{1}(G, F)=0$ for all $G \in \mathrm{fp}\left(\mathcal{F}^{\mathrm{op}}, \mathrm{Ab}\right)$. A functor $F$ : $\mathcal{F}^{\mathrm{op}} \rightarrow \mathrm{Ab}$ is fp-injective if and only if it is exact (cf. Lemma 2.7 in [17]) and therefore the restricted Yoneda functor

$$
\mathcal{S} \rightarrow\left(\mathcal{F}^{\mathrm{op}}, \mathrm{Ab}\right), \quad X \mapsto H_{X}=\left.\operatorname{Hom}(-, X)\right|_{\mathcal{F}},
$$

identifies the objects in $\mathcal{S}$ with the fp-injective objects in $\left(\mathcal{F}^{\mathrm{op}}, \mathrm{Ab}\right)$ since we assume Brown representability. Now let $\mathcal{C}^{\prime}=\left\{H_{X} \mid X \in \mathcal{C}\right\}$ and put

$$
\mathcal{T}^{\prime}=\left\{F \in \operatorname{fp}\left(\mathcal{F}^{\mathrm{op}}, \mathrm{Ab}\right) \mid \operatorname{Hom}(F, X)=0 \text { for all } X \in \mathcal{C}^{\prime}\right\} .
$$

Note that $\mathcal{T}=\left\{F^{\vee} \mid F \in \mathcal{T}^{\prime}\right\}$ since $F^{\vee}(X)=\operatorname{Hom}\left(F, H_{X}\right)$ for $X \in \mathcal{S}$. A reduced product of a family $\left(X_{i}\right)_{i \in I}$ of objects in $\left(\mathcal{F}^{\mathrm{op}}, \mathrm{Ab}\right)$ with respect to some filter $\mathcal{U}$ on $I$ is by definition the filtered colimit $\operatorname{colim}_{J \in \mathcal{U}} \prod_{i \in J} X_{i}$ so that the restricted Yoneda functor preserves reduced products. It follows from Proposition 4.5 of [16] that an fp-injective object $X$ in $\left(\mathcal{F}^{\mathrm{op}}, \mathrm{Ab}\right)$ is a subobject of some reduced product of objects in $\mathcal{C}^{\prime}$ if and only if $\operatorname{Hom}(F, X)=0$ for all $F \in \mathcal{T}^{\prime}$. Using again the restricted Yoneda functor, we deduce that $X \in \mathcal{S}$ fits into a triangle $X \rightarrow Y \rightarrow Z \rightarrow \Sigma X$ such that $Y$ is a reduced product of objects in $\mathcal{C}$ if and only if $F(X)$ for all $F \in \mathcal{T}$. This completes the proof since $\operatorname{Def} \mathcal{C}=\{X \in \mathcal{S} \mid F(X)=0$ for all $F \in \mathcal{T}\}$.

\section{Appendix: Finitely presented modules versus compact objects.}

In this appendix we explain the analogy between compact objects in a compactly generated triangulated category, and finitely presented modules in the category of modules over an associative ring. 
Let $\mathcal{A}$ be an additive category and suppose that it has arbitrary products and coproducts. We make the following definitions:

- An object $Q$ is $p$-injective if for every set $I$ the summation map $\coprod_{I} Q$ $\rightarrow Q$ factors through the canonical map $\coprod_{I} Q \rightarrow \prod_{I} Q$.

- A sequence of maps $X \rightarrow Y \rightarrow Z$ is p-exact if for every p-injective object $Q$ in $\mathcal{A}$ the sequence $0 \rightarrow \operatorname{Hom}(Z, Q) \rightarrow \operatorname{Hom}(Y, Q) \rightarrow \operatorname{Hom}(X, Q)$ $\rightarrow 0$ is exact.

- An object $P$ is p-projective if for every p-exact sequence $X \rightarrow Y \rightarrow Z$ the sequence $0 \rightarrow \operatorname{Hom}(P, X) \rightarrow \operatorname{Hom}(P, Y) \rightarrow \operatorname{Hom}(P, Z) \rightarrow 0$ is exact.

- An object $X$ is compact if the functor $\operatorname{Hom}(X,-)$ preserves coproducts.

If $\mathcal{A}$ is the category Mod $\Lambda$ of modules over an associative ring $\Lambda$, then the above concept of p-exactness coincides with the concept of pure-exactness introduced by Cohn [7]. This follows essentially from the characterization of pure-injective modules via the summation map which is due to Jensen and Lenzing (cf. [14, Proposition 7.32]). In this context the compact p-projective objects are characterized as follows.

Proposition. Let $\mathcal{A}$ be the category of modules over an associative ring. Then an object in $\mathcal{A}$ is compact and p-projective if and only if it is a finitely presented module.

Proof. The assertion is an immediate consequence of the well known fact that a module is pure-projective if and only if it is a direct factor of a coproduct of finitely presented modules.

Now suppose that $\mathcal{A}$ is a compactly generated triangulated category and denote by $\mathcal{F}$ the full subcategory of compact objects. Then the p-injective objects are precisely the objects which are pure-injective in the sense of Definition 1.1 (cf. [17, Theorem 1.8]). Therefore the functor $\mathcal{A} \rightarrow\left(\mathcal{F}^{\mathrm{op}}, \mathrm{Ab}\right)$, $X \mapsto H_{X}$, identifies the p-injective objects in $\mathcal{A}$ with the injective objects in $\left(\mathcal{F}^{\mathrm{op}}, \mathrm{Ab}\right)\left(\right.$ cf. $\left[17\right.$, Corollary 1.9]). Note that $\operatorname{Hom}(X, Q) \cong \operatorname{Hom}\left(H_{X}, H_{Q}\right)$ for all $X$ in $\mathcal{A}$ and every p-injective object $Q$ by Lemma 1.3. Therefore $X \mapsto H_{X}$ identifies the p-exact sequences $X \rightarrow Y \rightarrow Z$ with the exact sequences $0 \rightarrow H_{X} \rightarrow H_{Y} \rightarrow H_{Z} \rightarrow 0$ since the injective objects cogenerate $\left(\mathcal{F}^{\mathrm{op}}, \mathrm{Ab}\right)$.

Proposition. Let $\mathcal{A}$ be a compactly generated triangulated category. Then an object in $\mathcal{A}$ is compact and p-projective if and only if it is compact.

Proof. We need to show that every compact object is p-projective. However, this is just a reformulation of the fact that for each p-exact sequence $X \rightarrow Y \rightarrow Z$ the sequence $0 \rightarrow H_{X} \rightarrow H_{Y} \rightarrow H_{Z} \rightarrow 0$ is exact. 


\section{References}

[1] J. F. Adams, A variant of E. H. Brown's representability theorem, Topology 10 (1971), 185-198.

[2] M. Auslander, Coherent functors, in: Proc. Conf. on Categorical Algebra (La Jolla, CA, 1965), Springer, 1966, 189-231.

[3] - Functors and morphisms determined by objects, in: Representation Theory of Algebras (Philadelphia, 1976), R. Gordon (ed.), Dekker, New York, 1978, 1-244.

[4] A. Beligiannis, Relative homological algebra and purity in triangulated categories, J. Algebra 227 (2000), 268-361.

[5] J. D. Christensen, B. Keller and A. Neeman, Failure of Brown representability in derived categories, Topology 40 (2001), 1331-1361.

[6] J. D. Christensen and N. P. Strickland, Phantom maps and homology theories, ibid. 37 (1998), 339-364.

[7] P. M. Cohn, On the free product of associative rings, Math. Z. 71 (1959), 380-398.

[8] W. W. Crawley-Boevey, Infinite dimensional modules in the representation theory of finite dimensional algebras, in: Algebras and Modules, I (Trondheim, 1996), CMS Conf. Proc. 23, Amer. Math. Soc., 1998, 29-54.

[9] P. Freyd, Stable homotopy, in: Proc. Conf. on Categorical Algebra (La Jolla, CA, 1965), Springer, 1966, 121-172.

[10] -, Stable homotopy II, in: Proc. Sympos. Pure Math. 17, Amer. Math. Soc., 1970, 161-183.

[11] P. Gabriel, Des catégories abéliennes, Bull. Soc. Math. France 90 (1962), 323-448.

[12] I. Herzog, The Ziegler spectrum of a locally coherent Grothendieck category, Proc. London Math. Soc. 74 (1997), 503-558.

[13] M. Hovey, J. H. Palmieri and N. P. Strickland, Axiomatic stable homotopy theory, Mem. Amer. Math. Soc. 610 (1997).

[14] C. U. Jensen and H. Lenzing, Model Theoretic Algebra, Gordon and Breach, New York, 1989.

[15] H. Krause, The spectrum of a locally coherent category, J. Pure Appl. Algebra 114 (1997), 259-271.

[16] -, Exactly definable categories, J. Algebra 201 (1998), 456-492.

[17] —, Smashing subcategories and the telescope conjecture-an algebraic approach, Invent. Math. 139 (2000), 99-133.

[18] H. Krause and U. Reichenbach, Endofiniteness in stable homptopy theory, Trans. Amer. Math. Soc. 353 (2001), 157-173.

[19] H. R. Margolis, Spectra and the Steenrod Algebra, North-Holland, 1983.

[20] B. Mitchell, Rings with several objects, Adv. Math. 8 (1972), 1-161.

[21] O. Oberst and H. Rohrl, Flat and coherent functors, J. Algebra 14 (1970), 91-105.

[22] B. Stenstrøm, Rings of Quotients, Springer, 1975.

[23] M. Ziegler, Model theory of modules, Ann. Pure Appl. Logic 26 (1984), 149-213.

Fakultät für Mathematik

Universität Bielefeld

33501 Bielefeld, Germany

E-mail: henning@mathematik.uni-bielefeld.de

Received 16 February 2001;

in revised form 15 October 2001 